\title{
Barnes-type Narumi polynomials
}

Dae San Kim ${ }^{1}$ and Taekyun Kim²

"Correspondence: tkkim@kw.ac.kr ${ }^{2}$ Department of Mathematics, Kwangwoon University, Seoul, 139-701, Republic of Korea Full list of author information is available at the end of the article

\section{Abstract}

In this paper, we study the Barnes-type Narumi polynomials with umbral calculus viewpoint. From our study, we derive various identities of the Barnes-type Narumi polynomials.

MSC: 05A19; 05A40; 11B68

Keywords: Barnes-type Narumi polynomial; umbral calculus

\section{Introduction}

As is well known, the Narumi polynomials of order $\alpha$ are defined by the generating function to be

$$
\left(\frac{t}{\log (1+t)}\right)^{\alpha}(1+t)^{x}=\sum_{n=0}^{\infty} N_{n}^{(\alpha)}(x) \frac{t^{n}}{n !} \quad(\text { see [1]). }
$$

Let $r \in \mathbb{Z}_{>0}$. We consider the polynomials $N_{n}\left(x \mid a_{1}, \ldots, a_{r}\right)$ and $\hat{N}_{n}\left(x \mid a_{1}, \ldots, a_{r}\right)$, respectively, called the Barnes-type Narumi polynomials of the first kind and those of the second kind and respectively given by

$$
\prod_{j=1}^{r}\left(\frac{(1+t)^{a_{j}}-1}{\log (1+t)}\right)(1+t)^{x}=\sum_{n=0}^{\infty} N_{n}\left(x \mid a_{1}, \ldots, a_{r}\right) \frac{t^{n}}{n !}
$$

and

$$
\prod_{j=1}^{r}\left(\frac{(1+t)^{a_{j}}-1}{\log (1+t)(1+t)^{a_{j}}}\right)(1+t)^{x}=\sum_{n=0}^{\infty} \hat{N}_{n}\left(x \mid a_{1}, \ldots, a_{r}\right) \frac{t^{n}}{n !},
$$

where $a_{1}, a_{2}, \ldots, a_{r} \neq 0$.

When $x=0$,

$$
N_{n}\left(a_{1}, \ldots, a_{r}\right)=N_{n}\left(0 \mid a_{1}, \ldots, a_{r}\right)
$$

and

$$
\hat{N}_{n}\left(a_{1}, \ldots, a_{r}\right)=\hat{N}_{n}\left(0 \mid a_{1}, \ldots, a_{r}\right)
$$

are respectively called the Barnes-type Narumi numbers of the first kind and those of the second kind.

\section{焦 Springer}

○2014 Kim and Kim; licensee Springer. This is an Open Access article distributed under the terms of the Creative Commons Attribution License (http://creativecommons.org/licenses/by/2.0), which permits unrestricted use, distribution, and reproduction in any medium, provided the original work is properly cited. 
Note that

$$
\begin{aligned}
& N_{n}(x \mid \underbrace{1, \ldots, 1}_{r})=N_{n}^{(r)}(x), \\
& \hat{N}_{n}(x \mid \underbrace{1, \ldots, 1}_{r})=\hat{N}_{n}^{(r)}(x)
\end{aligned}
$$

and

$$
\hat{N}_{n}(x \mid \underbrace{1, \ldots, 1}_{r})=N_{n}^{(r)}(x-r) .
$$

In the previous paper [2], $N_{n}^{(\alpha)}(x)$ was denoted by $N_{n}^{(-\alpha)}$ and called the Narumi polynomial of order $\alpha$.

The Bernoulli polynomials are defined by the generating function to be

$$
\frac{t}{e^{t}-1} e^{x t}=\sum_{n=0}^{\infty} B_{n}(x) \frac{t^{n}}{n !} \quad(\text { see }[3-6])
$$

When $x=0, B_{n}=B_{n}(0)$ are called the Bernoulli numbers. In [7], it is known that the Cauchy numbers are given by

$$
\frac{t}{\log (1+t)}=\sum_{n=0}^{\infty} C_{n} \frac{t^{n}}{n !}
$$

It is well known that the Stirling number of the first kind is given by

$$
(x)_{n}=x(x-1) \cdots(x-n+1)=\sum_{l=0}^{\infty} S_{1}(n, l) x^{l} \quad(n \geq 0)(\text { see }[1,2,7-11])
$$

From (6), we have

$$
(\log (1+t))^{n}=n ! \sum_{l=n}^{\infty} S_{1}(l, n) \frac{t^{l}}{l !} \quad(n \geq 0)
$$

Let $\mathbb{C}$ be the complex number field and let $\mathcal{F}$ be the set of all formal power series in the variable $t$ :

$$
\mathcal{F}=\left\{f(t)=\sum_{k=0}^{\infty} a_{k} \frac{t^{k}}{k !} \mid a_{k} \in \mathbb{C}\right\} .
$$

Let $\mathbb{P}=\mathbb{C}[x]$ and let $\mathbb{P}^{*}$ be the vector space of all linear functionals on $\mathbb{P} .\langle L \mid p(x)\rangle$ denotes the action of the linear functional $L$ on $p(x)$ which satisfies $\langle L+M \mid p(x)\rangle=\langle L \mid p(x)\rangle+$ $\langle M \mid p(x)\rangle$, and $\langle c L \mid p(x)\rangle=c\langle L \mid p(x)\rangle$, where $c$ is a complex constant. The linear functional $\langle f(t) \mid \cdot\rangle$ on $\mathbb{P}$ is defined by $\left\langle f(t) \mid x^{n}\right\rangle=a_{n}(n \geq 0)$, where $f(t) \in \mathcal{F}$. Thus, we note that

$$
\left\langle t^{k} \mid x^{n}\right\rangle=n ! \delta_{n, k} \quad(n, k \geq 0)
$$

where $\delta_{n, k}$ is the Kronecker symbol (see [12-18]). 
For $f_{L}(t)=\sum_{k=0}^{\infty} \frac{\left\langle L \mid x^{k}\right\rangle}{k !} t^{k}$, we have $\left\langle f_{L}(t) \mid x^{n}\right\rangle=\left\langle L \mid x^{n}\right\rangle$. So, the map $L \mapsto f_{L}(t)$ is a vector space isomorphism from $\mathbb{P}^{*}$ onto $\mathcal{F}$. Henceforth, $\mathcal{F}$ denotes both the algebra of formal power series in $t$ and the vector space of all linear functionals on $\mathbb{P}$, and so an element $f(t)$ of $\mathcal{F}$ will be thought of as both a formal power series and a linear functional. We call $\mathcal{F}$ the umbral algebra. The order $o(f(t))$ of a power series $f(t) \neq 0$ is the smallest integer for which the coefficient of $t^{k}$ does not vanish. If $o(f(t))=1$, then $f(t)$ is called a delta series; if $o(f(t))=0$, then $g(t)$ is called an invertible series. Let $f(t), g(t) \in \mathcal{F}$ with $o(f(t))=1$ and $o(g(t))=0$. Then there exists a unique sequence $s_{n}(x)\left(\operatorname{deg} s_{n}(x)=n\right)$ such that $\left\langle g(t) f(t)^{k} \mid s_{n}(x)\right\rangle=n ! \delta_{n, k}$ for $n, k \geq 0$. The sequence $s_{n}(x)$ is called the Sheffer sequence for $(g(t), f(t))$ which is denoted by $s_{n}(x) \sim(g(t), f(t))$.

For $f(t), g(t) \in \mathcal{F}$ and $p(x) \in \mathbb{P}$, we have

$$
\langle f(t) g(t) \mid p(x)\rangle=\langle f(t) \mid g(t) p(x)\rangle=\langle g(t) \mid f(t) p(x)\rangle
$$

and

$$
\begin{aligned}
& f(t)=\sum_{k=0}^{\infty}\left\langle f(t) \mid x^{k}\right\rangle \frac{t^{k}}{k !}, \\
& p(x)=\sum_{k=0}^{\infty}\left\langle t^{k} \mid p(x)\right\rangle \frac{x^{k}}{k !} .
\end{aligned}
$$

From (10), we can derive the following equation (11):

$$
t^{k} p(x)=p^{(k)}(x)=\frac{d^{k} p(x)}{d x^{k}}, \quad e^{y t} p(x)=p(x+y) \quad(\text { see [1]) }
$$

Let $s_{n}(x) \sim(g(t), f(t))$. Then the following will be used:

$$
\frac{d s_{n}(x)}{d x}=\sum_{l=0}^{n-1}\left(\begin{array}{l}
n \\
l
\end{array}\right)\left\langle\bar{f}(t) \mid x^{n-l}\right\rangle s_{l}(x)
$$

where $\bar{f}(t)$ is the compositional inverse of $f(t)$ with $\bar{f}(f(t))=f(\bar{f}(t))=t$,

$$
\begin{aligned}
& \frac{1}{g(\bar{f}(t))} e^{x \bar{f}(t)}=\sum_{n=0}^{\infty} s_{n}(x) \frac{t^{n}}{n !} \quad \text { for all } x \in \mathbb{C}, \\
& f(t) s_{n}(x)=n s_{n-1}(x) \quad(n \geq 1), \quad s_{n}(x)=\sum_{j=0}^{n} \frac{\left\langle g(\bar{f}(t))^{-1} \bar{f}(t)^{j} \mid x^{n}\right\rangle}{j !} x^{j}, \\
& s_{n}(x+y)=\sum_{j=0}^{n}\left(\begin{array}{l}
n \\
j
\end{array}\right) s_{j}(x) p_{n-j}(y), \quad \text { where } p_{n}(x)=g(t) s_{n}(x), \\
& \langle f(t) \mid x p(x)\rangle=\left\langle\partial_{t} f(t) \mid p(x)\right\rangle, \quad \text { where } \partial_{t} f(t)=\frac{d f(t)}{d t}
\end{aligned}
$$

and

$$
s_{n+1}(x)=\left(x-\frac{g^{\prime}(t)}{g(t)}\right) \frac{1}{f^{\prime}(t)} s_{n}(x) \quad(n \geq 0)(\text { see }[1,19]) .
$$


Let us assume that $s_{n}(x) \sim(g(t), f(t))$ and $r_{n}(x) \sim(h(t), l(t))$. Then we have

$$
s_{n}(x)=\sum_{m=0}^{n} c_{n, m} r_{m}(x) \quad(n \geq 0)
$$

where

$$
c_{n, m}=\frac{1}{m !}\left\langle\frac{h(\bar{f}(t))}{g(\bar{f}(t))} l(\bar{f}(t))^{m} \mid x^{n}\right\rangle \quad(\text { see }[1,5]) .
$$

From (2), (3) and (13), we note that

$$
N_{n}\left(x \mid a_{1}, \ldots, a_{r}\right) \sim\left(\prod_{j=1}^{r}\left(\frac{t}{e^{a_{j} t}-1}\right), e^{t}-1\right)
$$

and

$$
\hat{N}_{n}\left(x \mid a_{1}, \ldots, a_{r}\right) \sim\left(\prod_{j=1}^{r}\left(\frac{t e^{a_{j} t}}{e^{a_{j} t}-1}\right), e^{t}-1\right) .
$$

In this paper, we study the Barnes-type Narumi polynomials with umbral calculus viewpoint. From our study, we derive various identities of the Barnes-type Narumi polynomials.

\section{Barnes-type Narumi polynomials}

From (21), we note that

$$
\prod_{j=1}^{r}\left(\frac{t}{e^{a_{j} t}-1}\right) N_{n}\left(x \mid a_{1}, \ldots, a_{r}\right) \sim\left(1, e^{t}-1\right)
$$

and

$$
(x)_{n} \sim\left(1, e^{t}-1\right) .
$$

Thus, by (22) and (23), we get

$$
\begin{aligned}
N_{n}\left(x \mid a_{1}, \ldots, a_{r}\right) & =\prod_{j=1}^{r}\left(\frac{e^{a_{j} t}-1}{t}\right)(x)_{n} \\
& =\sum_{m=0}^{n} S_{1}(n, m) \prod_{j=1}^{r}\left(\frac{e^{a_{j} t}-1}{t}\right) x^{m} .
\end{aligned}
$$

Note that

$$
\begin{aligned}
\prod_{j=1}^{r}\left(\frac{e^{a_{j} t}-1}{t}\right) & =\left(\sum_{l_{1}=0}^{\infty} \frac{a_{1}^{l_{1}+1}}{\left(l_{1}+1\right) !} t^{l_{1}}\right) \times \cdots \times\left(\sum_{l_{r}=0}^{\infty} \frac{a_{r}^{l_{r}+1}}{\left(l_{r}+1\right) !} t^{l_{r}}\right) \\
& =\sum_{l_{1}, \ldots, l_{r}=0}^{\infty} \sum_{l_{1}+\cdots+l_{r}=i} \frac{a_{1}^{l_{1}+1} \cdots a_{r}^{l_{r}+1}}{\left(l_{1}+1\right) ! \cdots\left(l_{r}+1\right) !} t^{i} .
\end{aligned}
$$


From (24) and (25), we have

$$
\begin{aligned}
& N_{n}\left(x \mid a_{1}, \ldots, a_{r}\right)=\sum_{m=0}^{\infty} S_{1}(n, m) \sum_{i=0}^{m} \sum_{l_{1}+\cdots+l_{r}=i} \frac{a_{1}^{l_{1}+1} \cdots a_{r}^{l_{r}+1}}{\left(l_{1}+1\right) ! \cdots\left(l_{r}+1\right) !} t^{i} x^{m} \\
& =\sum_{m=0}^{n} S_{1}(n, m) \sum_{i=0}^{m} \frac{i !}{(i+r) !} \sum_{l_{1}+\cdots+l_{r}=i}\left(\begin{array}{c}
i+r \\
l_{1}+1, \ldots, l_{r}+1
\end{array}\right)\left(\begin{array}{c}
m \\
i
\end{array}\right) \\
& \times a_{1}^{l_{1}+1} \cdots a_{r}^{l_{r}+1} x^{m-i} \\
& =\sum_{i=0}^{n}\left\{\sum_{m=i}^{n} \sum_{l_{1}+\cdots+l_{r}=m-i} S_{1}(n, m) \frac{(m-i) !}{(m-i+r) !}\right. \\
& \left.\times\left(\begin{array}{c}
m-i+r \\
l_{1}+1, \ldots, l_{r}+1
\end{array}\right)\left(\begin{array}{c}
m \\
i
\end{array}\right) a_{1}^{l_{1}+1} \cdots a_{r}^{l_{r}+1}\right\} x^{i} .
\end{aligned}
$$

By (21), we see that

$$
\prod_{j=1}^{r}\left(\frac{t e^{a_{j} t}}{e^{a_{j} t}-1}\right) \hat{N}_{n}\left(x \mid a_{1}, \ldots, a_{r}\right) \sim\left(1, e^{t}-1\right),
$$

and we recall (23)

Thus, we have

$$
\begin{aligned}
& \hat{N}_{n}\left(x \mid a_{1}, \ldots, a_{r}\right)=\prod_{j=1}^{r}\left(\frac{e^{a_{j} t}-1}{t e^{a_{j} t}}\right)(x)_{n}=e^{-\sum_{j=1}^{r} a_{j} t} \prod_{j=1}^{r}\left(\frac{e^{a_{j} t}-1}{t}\right)(x)_{n} \\
& =e^{-\sum_{j=1}^{r} a_{j} t} N_{n}\left(x \mid a_{1}, \ldots, a_{r}\right) \\
& =\sum_{i=0}^{n}\left\{\sum_{m=i}^{n} \sum_{l_{1}+\cdots+l_{r}=m-i} S_{1}(n, m) \frac{(m-i) !}{(m-i+r) !}\right. \\
& \left.\times\left(\begin{array}{c}
m-i+r \\
l_{1}+1, \ldots, l_{r}+1
\end{array}\right)\left(\begin{array}{c}
m \\
i
\end{array}\right) a_{1}^{l_{1}+1} \cdots a_{r}^{l_{r}+1}\right\} e^{-\sum_{j=1}^{r} a_{j} t} x^{i} \\
& =\sum_{i=0}^{n}\left\{\sum_{m=i}^{n} \sum_{l_{1}+\cdots+l_{r}=m-i} S_{1}(n, m) \frac{(m-i) !}{(m-i+r) !}\right. \\
& \left.\times\left(\begin{array}{c}
m-i+r \\
l_{1}+1, \ldots, l_{r}+1
\end{array}\right)\left(\begin{array}{c}
m \\
i
\end{array}\right) a_{1}^{l_{1}+1} \cdots a_{r}^{l_{r}+1}\right\}\left(x-\sum_{j=1}^{r} a_{j}\right)^{i}
\end{aligned}
$$

or

$$
\begin{aligned}
\hat{N}_{n}\left(x \mid a_{1}, \ldots, a_{r}\right)= & \prod_{j=1}^{r}\left(\frac{e^{a_{j} t}-1}{t e^{a_{j} t}}\right)(x)_{n}=\prod_{j=1}^{r}\left(\frac{e^{-a_{j} t}-1}{-t}\right)(x)_{n} \\
= & \sum_{m=0}^{n} S_{1}(n, m) \sum_{i=0}^{m}(-1)^{i} \frac{i !}{(i+r) !} \\
& \times \sum_{l_{1}+\cdots+l_{r}=i}\left(\begin{array}{c}
i+r \\
i_{1}+1, \ldots, i_{r}+1
\end{array}\right)\left(\begin{array}{c}
m \\
i
\end{array}\right) a_{1}^{l_{1}+1} \cdots a_{r}^{l_{r}+1} x^{m-i}
\end{aligned}
$$




$$
\begin{aligned}
& =\sum_{i=0}^{n}\left\{\sum_{m=i}^{n} \sum_{l_{1}+\cdots+l_{r}=m-i}(-1)^{m-i} S_{1}(n, m) \frac{(m-i) !}{(m-i+r) !}\right. \\
& \left.\times\left(\begin{array}{c}
m-i+r \\
l_{1}+1, \ldots, l_{r}+1
\end{array}\right)\left(\begin{array}{c}
m \\
i
\end{array}\right) a_{1}^{l_{1}+1} \cdots a_{r}^{l_{r}+1}\right\} x^{i} .
\end{aligned}
$$

Therefore, by (26), (28) and (29), we obtain the following theorem.

Theorem 1 For $n \geq 0$, we have

$$
\begin{aligned}
& N_{n}\left(x \mid a_{1}, \ldots, a_{r}\right)=\sum_{i=0}^{n}\left\{\sum_{m=i}^{n} \sum_{l_{1}+\cdots+l_{r}=m-i} S_{1}(n, m) \frac{(m-i) !}{(m-i+r) !}\right. \\
& \left.\times\left(\begin{array}{c}
m-i+r \\
l_{1}+1, \ldots, l_{r}+1
\end{array}\right)\left(\begin{array}{c}
m \\
i
\end{array}\right) a_{1}^{l_{1}+1} \cdots a_{r}^{l_{r}+1}\right\} x^{i}
\end{aligned}
$$

and

$$
\begin{aligned}
& \hat{N}_{n}\left(x \mid a_{1}, \ldots, a_{r}\right)=\sum_{i=0}^{n}\left\{\sum_{m=i}^{n} \sum_{l_{1}+\cdots+l_{r}=m-i} S_{1}(n, m) \frac{(m-i) !}{(m-i+r) !}\right. \\
& \left.\times\left(\begin{array}{c}
m-i+r \\
l_{1}+1, \ldots, l_{r}+1
\end{array}\right)\left(\begin{array}{c}
m \\
i
\end{array}\right) a_{1}^{l_{1}+1} \cdots a_{r}^{l_{r}+1}\right\}\left(x-\sum_{j=1}^{r} a_{j}\right)^{i} \\
& =\sum_{i=0}^{n}\left\{\sum_{m=i}^{n} \sum_{l_{1}+\cdots+l_{r}=m-i}(-1)^{m-i} S_{1}(n, m)\right. \\
& \left.\times \frac{(m-i) !}{(m-i+r) !}\left(\begin{array}{c}
m-i+r \\
l_{1}+1, \ldots, l_{r}+1
\end{array}\right)\left(\begin{array}{c}
m \\
j
\end{array}\right) a_{1}^{l_{1}+1} \cdots a_{r}^{l_{r}+1}\right\} x^{i} .
\end{aligned}
$$

From (14), we can derive the following equation (30):

$$
N_{n}\left(x \mid a_{1}, \ldots, a_{r}\right)=\sum_{j=0}^{n} \frac{1}{j !}\left\langle\prod_{j=1}^{r}\left(\frac{(1+t)^{a_{j}}-1}{\log (1+t)}\right)(\log (1+t))^{j} \mid x^{n}\right\rangle x^{j}
$$

where

$$
\begin{aligned}
& \left\langle\prod_{j=1}^{r}\left(\frac{(1+t)^{a_{j}}-1}{\log (1+t)}\right)(\log (1+t))^{j} \mid x^{n}\right\rangle \\
& =\left\langle\prod_{j=1}^{r}\left(\frac{(1+t)^{a_{j}}-1}{\log (1+t)}\right) \mid(\log (1+t))^{j} x^{n}\right\rangle \\
& =\left\langle\prod_{j=1}^{r}\left(\frac{(1+t)^{a_{j}}-1}{\log (1+t)}\right) \mid j ! \sum_{l=j}^{\infty} S_{1}(l, j) \frac{t^{l}}{l !} x^{n}\right\rangle \\
& =j ! \sum_{l=j}^{n}\left(\begin{array}{l}
n \\
l
\end{array}\right) S_{1}(l, j) N_{n-l}\left(a_{1}, \ldots, a_{r}\right) .
\end{aligned}
$$

Thus, by (30) and (31), we obtain the following theorem. 
Theorem 2 For $n \geq 0$, we have

$$
N_{n}\left(x \mid a_{1}, \ldots, a_{r}\right)=\sum_{j=0}^{n}\left\{\sum_{l=j}^{n}\left(\begin{array}{l}
n \\
l
\end{array}\right) S_{1}(l, j) N_{n-l}\left(a_{1}, \ldots, a_{r}\right)\right\} x^{j}
$$

By the same methods as in (28), (29) and (30), we get

$$
\begin{aligned}
& \hat{N}_{n}\left(x \mid a_{1}, \ldots, a_{r}\right) \\
& =\sum_{j=0}^{n}\left\{\sum_{l=j}^{n}\left(\begin{array}{l}
n \\
l
\end{array}\right) S_{1}(l, j) N_{n-l}\left(a_{1}, \ldots, a_{r}\right)\right\}\left(x-\sum_{i=1}^{r} a_{i}\right)^{j} \\
& =\sum_{j=0}^{n}\left\{\sum_{l=j}^{n}\left(\begin{array}{l}
n \\
l
\end{array}\right) S_{1}(l, j) \hat{N}_{n-l}\left(a_{1}, \ldots, a_{r}\right)\right\} x^{j} .
\end{aligned}
$$

By (8), we get

$$
\begin{aligned}
N_{n}\left(y \mid a_{1}, \ldots, a_{r}\right) & =\left\langle\sum_{i=0}^{\infty} N_{i}\left(y \mid a_{1}, \ldots, a_{r}\right) \frac{t^{i}}{i !} \mid x^{n}\right\rangle \\
& =\left\langle\prod_{j=1}^{r}\left(\frac{(1+t)^{a_{j}}-1}{\log (1+t)}\right)(1+t)^{y} \mid x^{n}\right\rangle \\
& =\left\langle\prod_{j=1}^{r}\left(\frac{(1+t)^{a_{j}}-1}{\log (1+t)}\right) \mid \sum_{m=0}^{\infty}(y)_{m} \frac{t^{m}}{m !} x^{m}\right\rangle \\
& =\sum_{m=0}^{n}(y)_{m}\left(\begin{array}{c}
n \\
m
\end{array}\right)\left\langle\prod_{j=1}^{r}\left(\frac{(1+t)^{a_{j}}-1}{\log (1+t)}\right) \mid x^{n-m}\right\rangle \\
& =\sum_{m=0}^{n}(y)_{m}\left(\begin{array}{c}
n \\
m
\end{array}\right) N_{n-m}\left(a_{1}, \ldots, a_{r}\right)
\end{aligned}
$$

and

$$
\begin{aligned}
\hat{N}_{n}\left(y \mid a_{1}, \ldots, a_{r}\right) & =\left\langle\sum_{i=0}^{\infty} \hat{N}_{i}\left(y \mid a_{1}, \ldots, a_{r}\right) \frac{t^{i}}{i !} \mid x^{n}\right\rangle \\
& =\left\langle\prod_{j=1}^{r}\left(\frac{(1+t)^{a_{j}}-1}{\log (1+t)(1+t)^{a_{j}}}\right)(1+t)^{y} \mid x^{n}\right\rangle \\
& =\left\langle\prod_{j=1}^{r}\left(\frac{(1+t)^{a_{j}}-1}{\log (1+t)(1+t)^{a_{j}}}\right) \mid \sum_{m=0}^{\infty}(y)_{m} \frac{t^{m}}{m !} x^{n}\right\rangle \\
& =\sum_{m=0}^{n}(y)_{m}\left(\begin{array}{c}
n \\
m
\end{array}\right)\left\langle\prod_{j=1}^{r}\left(\frac{(1+t)^{a_{j}}-1}{\log (1+t)(1+t)^{a_{j}}}\right) \mid x^{n-m}\right\rangle \\
& =\sum_{m=0}^{n}\left(\begin{array}{c}
n \\
m
\end{array}\right)(y)_{m} \hat{N}_{n-m}\left(a_{1}, \ldots, a_{r}\right) .
\end{aligned}
$$

Therefore, by (33) and (34), we obtain the following theorem. 
Theorem 3 For $n \geq 0$, we have

$$
N_{n}\left(x \mid a_{1}, \ldots, a_{r}\right)=\sum_{m=0}^{n}\left(\begin{array}{l}
n \\
m
\end{array}\right) N_{n-m}\left(a_{1}, \ldots, a_{r}\right)(x)_{m}
$$

and

$$
\hat{N}_{n}\left(x \mid a_{1}, \ldots, a_{r}\right)=\sum_{m=0}^{n}\left(\begin{array}{l}
n \\
m
\end{array}\right) \hat{N}_{n-m}\left(a_{1}, \ldots, a_{r}\right)(x)_{m} .
$$

From (15), we note that

$$
N_{n}\left(x+y \mid a_{1}, \ldots, a_{r}\right)=\sum_{j=0}^{n}\left(\begin{array}{l}
n \\
j
\end{array}\right) N_{j}\left(x \mid a_{1}, \ldots, a_{r}\right)(y)_{n-j}
$$

and

$$
\hat{N}_{n}\left(x+y \mid a_{1}, \ldots, a_{r}\right)=\sum_{j=0}^{n}\left(\begin{array}{l}
n \\
j
\end{array}\right) \hat{N}_{j}\left(x \mid a_{1}, \ldots, a_{r}\right)(y)_{n-j} .
$$

By (14), we get

$$
\left(e^{t}-1\right) N_{n}\left(x \mid a_{1}, \ldots, a_{r}\right)=n N_{n-1}\left(x \mid a_{1}, \ldots, a_{r}\right)
$$

and

$$
\begin{aligned}
\left(e^{t}-1\right) N_{n}\left(x \mid a_{1}, \ldots, a_{r}\right) & =e^{t} N_{n}\left(x \mid a_{1}, \ldots, a_{r}\right)-N_{n}\left(x \mid a_{1}, \ldots, a_{r}\right) \\
& =N_{n}\left(x+1 \mid a_{1}, \ldots, a_{r}\right)-N\left(x \mid a_{1}, \ldots, a_{r}\right) .
\end{aligned}
$$

From (37) and (38), we have

$$
N_{n}\left(x+1 \mid a_{1}, \ldots, a_{r}\right)-N_{n}\left(x \mid a_{1}, \ldots, a_{r}\right)=n N_{n-1}\left(x \mid a_{1}, \ldots, a_{r}\right) .
$$

By the same method as (39), we get

$$
\hat{N}_{n}\left(x+1 \mid a_{1}, \ldots, a_{r}\right)-\hat{N}_{n}\left(x \mid a_{1}, \ldots, a_{r}\right)=n \hat{N}_{n-1}\left(x \mid a_{1}, \ldots, a_{r}\right) .
$$

Recall that $N_{n}\left(x \mid a_{1}, \ldots, a_{r}\right) \sim\left(\prod_{j=1}^{r}\left(\frac{t}{e^{a_{j} t}-1}\right), e^{t}-1\right)$.

From (17), we can derive the following equation (41):

$$
N_{n+1}\left(x \mid a_{1}, \ldots, a_{r}\right)=x N_{n}\left(x-1 \mid a_{1}, \ldots, a_{r}\right)-e^{-t} \frac{g^{\prime}(t)}{g(t)} N_{n}\left(x \mid a_{1}, \ldots, a_{r}\right) .
$$

Now, we observe that

$$
\begin{aligned}
\frac{g^{\prime}(t)}{g(t)} & =(\log g(t))^{\prime}=\left(r \log t-\sum_{j=1}^{r} \log \left(e^{a_{j} t}-1\right)\right)^{\prime}=\frac{r}{t}-\sum_{j=1}^{r} \frac{a_{j} e^{a_{j} t}}{e^{a_{j} t}-1} \\
& =\frac{\sum_{j=1}^{r} \prod_{i \neq j}^{r}\left(e^{a_{i} t}-1\right)\left\{e^{a_{j} t}-1-t a_{j} e^{a_{j} t}\right\}}{t \prod_{j=1}^{r}\left(e^{a_{j} t}-1\right)},
\end{aligned}
$$


where

$$
\begin{aligned}
r-\sum_{j=1}^{r} \frac{a_{j} t e^{a_{j} t}}{e^{a_{j} t}-1} & =\frac{\sum_{j=1}^{r} \prod_{i \neq j}\left(e^{a_{i} t}-1\right)\left\{e^{a_{j} t}-1-a_{j} t e^{a_{j} t}\right\}}{\prod_{j=1}^{r}\left(e^{a_{j} t}-1\right)} \\
& =\frac{-\frac{1}{2}\left(\sum_{j=1}^{r} a_{1} a_{2} \cdots a_{j-1} a_{j}^{2} a_{j+1} \cdots a_{r}\right) t^{r+1}+\cdots}{\left(a_{1} a_{2} \cdots a_{r}\right) t^{r}} \\
& =-\frac{1}{2}\left(\sum_{j=1}^{r} a_{j}\right) t+\cdots
\end{aligned}
$$

has at least the order 1 .

By (42) and (43), we get

$$
\begin{aligned}
\frac{g^{\prime}(t)}{g(t)} N_{n}\left(x \mid a_{1}, \ldots, a_{r}\right) & \frac{r-\sum_{j=1}^{r} \frac{a_{j} t t^{a_{j} t}}{e^{a_{j} t}-1}}{t}\left(\sum_{i=0}^{n}\left\{\sum_{l=i}^{n}\left(\begin{array}{c}
n \\
l
\end{array}\right) S_{1}(l, i) N_{n-l}\left(a_{1}, \ldots, a_{r}\right)\right\} x^{i}\right) \\
= & \sum_{i=0}^{n}\left\{\sum_{l=i}^{n}\left(\begin{array}{c}
n \\
l
\end{array}\right) S_{1}(l, i) N_{n-l}\left(a_{1}, \ldots, a_{r}\right)\right\} \frac{r-\sum_{j=1}^{r} \frac{a_{j} t e^{a_{j} t}}{e^{a_{j} t}-1}}{t} x^{i} \\
= & \sum_{i=0}^{n}\left\{\sum_{l=i}^{n}\left(\begin{array}{c}
n \\
l
\end{array}\right) S_{1}(l, i) N_{n-l}\left(a_{1}, \ldots, a_{r}\right)\right\}\left(r-\sum_{j=1}^{r} \frac{a_{j} t e^{a_{j} t}}{e^{a_{j} t}-1}\right) \frac{x^{i+1}}{i+1} \\
= & \sum_{i=0}^{n} \frac{1}{i+1}\left\{\sum_{l=i}^{n}\left(\begin{array}{c}
n \\
l
\end{array}\right) S_{1}(l, i) N_{n-l}\left(a_{1}, \ldots, a_{r}\right)\right\}\left(r x^{i+1}-\sum_{j=1}^{r} \sum_{m=0}^{\infty} B_{m} \frac{\left(-a_{j} t\right)^{m}}{m !} x^{i+1}\right) \\
= & -\sum_{i=0}^{n} \frac{1}{i+1}\left\{\sum_{l=i}^{n}\left(\begin{array}{c}
n \\
l
\end{array}\right) S_{1}(l, i) N_{n-l}\left(a_{1}, \ldots, a_{r}\right)\right\} \\
& \times \sum_{j=1}^{r} \sum_{m=1}^{i+1}(-1)^{m}\left(\begin{array}{c}
i+1 \\
m
\end{array}\right) B_{m} a_{j}^{m} x^{i+1-m} \\
= & -\sum_{i=0}^{n} \frac{1}{i+1}\left\{\sum_{l=i}^{n}\left(\begin{array}{c}
n \\
l
\end{array}\right) S_{1}(l, i) N_{n-l}\left(a_{1}, \ldots, a_{r}\right)\right\} \\
& \times \sum_{j=1}^{r} \sum_{m=0}^{i}(-1)^{i+1-m}\left(\begin{array}{c}
i+1 \\
m
\end{array}\right) a_{j}^{i+1-m} B_{i+1-m} x^{m} . \\
& \\
&
\end{aligned}
$$

Therefore, by (41) and (44), we obtain the following theorem.

Theorem 4 For $n \geq 0$, we have

$$
\begin{aligned}
& N_{n+1}\left(x \mid a_{1}, \ldots, a_{r}\right) \\
& =x N_{n}\left(x-1 \mid a_{1}, \ldots, a_{r}\right)+\sum_{m=0}^{n}\left\{\sum_{i=m}^{n} \sum_{l=i}^{n} \sum_{j=1}^{r} \frac{1}{i+1}\left(\begin{array}{c}
n \\
l
\end{array}\right)\left(\begin{array}{c}
i+1 \\
m
\end{array}\right) S_{1}(l, i)\right. \\
& \left.\quad \times B_{i+1-m}\left(-a_{j}\right)^{i+1-m} N_{n-l}\left(a_{1}, \ldots, a_{r}\right)\right\}(x-1)^{m} .
\end{aligned}
$$


By the same method as the proof of Theorem 4, we get

$$
\begin{aligned}
\hat{N}_{n+1}\left(x \mid a_{1}, \ldots, a_{r}\right)= & \left(x-\sum_{j=1}^{r} a_{j}\right) \hat{N}_{n}\left(x-1 \mid a_{1}, \ldots, a_{r}\right) \\
& +\sum_{m=0}^{n}\left\{\sum_{i=m}^{n} \sum_{l=i}^{n} \sum_{j=1}^{r} \frac{1}{i+1}\left(\begin{array}{c}
n \\
l
\end{array}\right)\left(\begin{array}{c}
i+1 \\
m
\end{array}\right) S_{1}(l, i)\right. \\
& \left.\times B_{i+1-m}\left(-a_{j}\right)^{i+1-m} \hat{N}_{n-l}\left(a_{1}, \ldots, a_{r}\right)\right\}(x-1)^{m} .
\end{aligned}
$$

From (12) and (20), we can derive the following equation (46):

$$
\left\langle\bar{f}(t) \mid x^{n-l}\right\rangle=\left\langle\log (1+t) \mid x^{n-l}\right\rangle=\left\langle\sum_{m=1}^{\infty} \frac{(-1)^{m-1}}{m} t^{m} \mid x^{n-l}\right\rangle=(-1)^{n-l-1}(n-l-1) ! .
$$

Thus, by (46), we get

$$
\begin{aligned}
\frac{d}{d x} N_{n}\left(x \mid a_{1}, \ldots, a_{r}\right) & =\sum_{l=0}^{n-1}\left(\begin{array}{l}
n \\
l
\end{array}\right)(-1)^{n-l-1}(n-l-1) ! N_{l}\left(x \mid a_{1}, \ldots, a_{r}\right) \\
& =n ! \sum_{l=0}^{n-1} \frac{(-1)^{n-l-1}}{l !(n-l)} N_{l}\left(x \mid a_{1}, \ldots, a_{r}\right) .
\end{aligned}
$$

By the same method as (47), we get

$$
\frac{d}{d x} \hat{N}_{n}\left(x \mid a_{1}, \ldots, a_{r}\right)=n ! \sum_{l=0}^{n-1} \frac{(-1)^{n-l-1}}{l !(n-l)} \hat{N}_{l}\left(x \mid a_{1}, \ldots, a_{r}\right) .
$$

From (8), we note that, for $n \geq 1$,

$$
\begin{aligned}
N_{n}\left(y \mid a_{1}, \ldots, a_{r}\right) & \\
= & \left\langle\sum_{i=0}^{\infty} N_{i}\left(y \mid a_{1}, \ldots, a_{r}\right) \frac{t^{i}}{i !} \mid x^{n}\right\rangle \\
= & \left\langle\prod_{j=1}^{r}\left(\frac{(1+t)^{a_{j}}-1}{\log (1+t)}\right)(1+t)^{y} \mid x^{n}\right\rangle \\
= & \left\langle\partial_{t}\left(\prod_{j=1}^{r}\left(\frac{(1+t)^{a_{j}}-1}{\log (1+t)}\right)(1+t)^{y}\right) \mid x^{n-1}\right\rangle \\
= & \left\langle\prod_{j=1}^{r}\left(\frac{(1+t)^{a_{j}}-1}{\log (1+t)}\right)\left(\partial_{t}(1+t)^{y}\right) \mid x^{n-1}\right\rangle \\
& +\left\langle\left(\partial_{t}\left(\frac{(1+t)^{a_{j}}-1}{\log (1+t)}\right)\right)(1+t)^{y} \mid x^{n-1}\right\rangle \\
= & y N_{n-1}\left(y-1 \mid a_{1}, \ldots, a_{r}\right)+\left\langle\left(\partial_{t}\left(\frac{(1+t)^{a_{j}}-1}{\log (1+t)}\right)\right)(1+t)^{y} \mid x^{n-1}\right\rangle .
\end{aligned}
$$


Now, we observe that

$$
\begin{aligned}
\partial_{t} & \prod_{j=1}^{r}\left(\frac{(1+t)^{a_{j}}-1}{\log (1+t)}\right) \\
& =\sum_{j=1}^{r} \prod_{i \neq j}\left(\frac{(1+t)^{a_{i}}-1}{\log (1+t)}\right) \frac{a_{j}(1+t)^{a_{j}-1} \log (1+t)-\left((1+t)^{a_{j}}-1\right) \frac{1}{1+t}}{(\log (1+t))^{2}} \\
& =\frac{1}{1+t} \prod_{i=1}^{r}\left(\frac{(1+t)^{a_{i}}-1}{\log (1+t)}\right) \sum_{j=1}^{r}\left\{\frac{a_{j}(1+t)^{a_{j}}}{(1+t)^{a_{j}}-1}-\frac{1}{\log (1+t)}\right\} \\
& =\frac{1}{1+t} \prod_{i=1}^{r}\left(\frac{(1+t)^{a_{i}}-1}{\log (1+t)}\right) \frac{\sum_{j=1}^{r}\left\{\frac{a_{j} t(1+t)^{a_{j}}}{(1+t)^{a_{j}}-1}-\frac{t}{t}\right\}}{\log (1+t)},
\end{aligned}
$$

where

$$
\sum_{j=1}^{r}\left\{\frac{a_{j} t(1+t)^{a_{j}}}{(1+t)^{a_{j}}-1}-\frac{t}{\log (1+t)}\right\}=\frac{1}{2}\left(\sum_{j=1}^{r} a_{j}\right) t+\cdots
$$

is a series with order greater than or equal to 1 .

By (50) and (51), we get

$$
\begin{aligned}
& \left\langle\left(\partial_{t} \prod_{j=1}^{r}\left(\frac{(1+t)^{a_{j}}-1}{\log (1+t)}\right)\right)(1+t)^{y} \mid x^{n-1}\right\rangle \\
& =\left\langle\prod_{i=1}^{r}\left(\frac{(1+t)^{a_{i}}-1}{\log (1+t)}\right) \frac{(1+t)^{y}}{1+t} \mid \frac{\sum_{j=1}^{r}\left\{\frac{a_{j} t(1+t)^{a_{j}}}{(1+t)^{a_{j}}-1}-\frac{t}{\log (1+t)}\right\}}{t} x^{n-1}\right\rangle \\
& =\frac{1}{n}\left\langle\prod_{i=1}^{r}\left(\frac{(1+t)^{a_{i}}-1}{\log (1+t)}\right)(1+t)^{y-1} \mid \sum_{j=1}^{r}\left\{\frac{a_{j} t(1+t)^{a_{j}}}{(1+t)^{a_{j}}-1}-\frac{t}{\log (1+t)}\right\} x^{n}\right\rangle \\
& =\frac{1}{n}\left\{\sum_{j=1}^{r} a_{j}\left\langle\frac{\log (1+t)}{(1+t)^{a_{j}}-1} \prod_{i=1}^{r}\left(\frac{(1+t)^{a_{i}}-1}{\log (1+t)}\right)(1+t)^{y+a_{j}-1} \mid \frac{t}{\log (1+t)} x^{n}\right\rangle\right. \\
& \left.-r\left\langle\prod_{i=1}^{r}\left(\frac{(1+t)^{a_{i}}-1}{\log (1+t)}\right)(1+t)^{y-1} \mid \frac{t}{\log (1+t)} x^{n}\right\rangle\right\} \\
& =\frac{1}{n} \sum_{j=1}^{r} a_{j} \sum_{l=0}^{n}\left(\begin{array}{l}
n \\
l
\end{array}\right) C_{l}\left\langle\frac{\log (1+t)}{(1+t)^{a_{j}}-1} \prod_{i=1}^{r}\left(\frac{(1+t)^{a_{i}}-1}{\log (1+t)}\right)(1+t)^{y+a_{j}-1} \mid x^{n-l}\right\rangle \\
& -\frac{r}{n} \sum_{l=0}^{n}\left(\begin{array}{l}
n \\
l
\end{array}\right) C_{l}\left\langle\prod_{i=1}^{r}\left(\frac{(1+t)^{a_{i}}-1}{\log (1+t)}\right)(1+t)^{y-1} \mid x^{n-l}\right\rangle \\
& =\frac{1}{n} \sum_{j=1}^{r} \sum_{l=0}^{n}\left(\begin{array}{l}
n \\
l
\end{array}\right) a_{j} C_{l} N_{n-l}\left(y+a_{j}-1 \mid a_{1}, \ldots, \hat{a}_{j}, \ldots, a_{r}\right) \\
& -\frac{r}{n} \sum_{l=0}^{n}\left(\begin{array}{l}
n \\
l
\end{array}\right) C_{l} N_{n-l}\left(y-1 \mid a_{1}, \ldots, a_{r}\right)
\end{aligned}
$$

where $\hat{a}_{j}$ means that $a_{j}$ is omitted. 
Therefore, by (49) and (52), we obtain the following theorem.

Theorem 5 For $n \geq 1$, we have

$$
\begin{aligned}
N_{n}\left(x \mid a_{1}, \ldots, a_{r}\right)= & x N_{n-1}\left(x-1 \mid a_{1}, \ldots, a_{r}\right) \\
& +\frac{1}{n} \sum_{j=1}^{r} \sum_{l=0}^{n}\left(\begin{array}{l}
n \\
l
\end{array}\right) a_{j} C_{l} N_{n-l}\left(x+a_{j}-1 \mid a_{1}, \ldots, \hat{a}_{j}, \ldots, a_{r}\right) \\
& -\frac{r}{n} \sum_{l=0}^{r}\left(\begin{array}{l}
n \\
l
\end{array}\right) C_{l} N_{n-l}\left(x-1 \mid a_{1}, \ldots, a_{r}\right),
\end{aligned}
$$

where $C_{n}$ are the Cauchy numbers with the generating function given by

$$
\frac{t}{\log (1+t)}=\sum_{n=0}^{\infty} C_{n} \frac{t^{n}}{n !}
$$

By the same method as the proof of Theorem 5, we get

$$
\begin{aligned}
\hat{N}_{n}\left(x \mid a_{1}, \ldots, a_{r}\right)= & \left(x-\sum_{j=1}^{r} a_{j}\right) \hat{N}_{n-1}\left(x-1 \mid a_{1}, \ldots, a_{r}\right) \\
& -\frac{r}{n} \sum_{l=0}^{n}\left(\begin{array}{l}
n \\
l
\end{array}\right) C_{l} \hat{N}_{n-l}\left(x-1 \mid a_{1}, \ldots, a_{r}\right) \\
& -\frac{1}{n} \sum_{j=1}^{r} \sum_{l=0}^{n}\left(\begin{array}{l}
n \\
l
\end{array}\right) a_{j} C_{l} \hat{N}_{n-l}\left(x-1 \mid a_{1}, \ldots, \hat{a}_{j}, \ldots, a_{r}\right) .
\end{aligned}
$$

Now we compute the following formula (54) in two different ways:

$$
\left\langle\prod_{j=1}^{r}\left(\frac{(1+t)^{a_{j}}-1}{\log (1+t)}\right)(\log (1+t))^{m} \mid x^{n}\right\rangle .
$$

On the one hand,

$$
\begin{aligned}
& \left\langle\prod_{j=1}^{r}\left(\frac{(1+t)^{a_{j}}-1}{\log (1+t)}\right)(\log (1+t))^{m} \mid x^{n}\right\rangle \\
& \quad=\left\langle\prod_{j=1}^{r}\left(\frac{(1+t)^{a_{j}}-1}{\log (1+t)}\right) \mid m ! \sum_{l=m}^{\infty} S_{1}(l, m) \frac{t^{l}}{l !} x^{n}\right\rangle \\
& \quad=m ! \sum_{l=m}^{n}\left(\begin{array}{l}
n \\
l
\end{array}\right) S_{1}(l, m)\left\langle\prod_{j=1}^{r}\left(\frac{(1+t)^{a_{j}}-1}{\log (1+t)}\right) \mid x^{n-l}\right\rangle \\
& \quad=m ! \sum_{l=m}^{n}\left(\begin{array}{l}
n \\
l
\end{array}\right) S_{1}(l, m) N_{n-l}\left(a_{1}, \ldots, a_{r}\right) \\
& \quad=m ! \sum_{l=0}^{n-m}\left(\begin{array}{l}
n \\
l
\end{array}\right) S_{1}(n-l, m) N_{l}\left(a_{1}, \ldots, a_{r}\right) .
\end{aligned}
$$


On the other hand,

$$
\begin{aligned}
& \left\langle\prod_{j=1}^{r}\left(\frac{(1+t)^{a_{j}}-1}{\log (1+t)}\right)(\log (1+t))^{m} \mid x^{n}\right\rangle \\
& =\left\langle\partial_{t}\left(\prod_{j=1}^{r}\left(\frac{(1+t)^{a_{j}}-1}{\log (1+t)}\right)(\log (1+t))^{m}\right) \mid x^{n-1}\right\rangle \\
& =\left\langle\prod_{j=1}^{r}\left(\frac{(1+t)^{a_{j}}-1}{\log (1+t)}\right)\left(\partial_{t}\left((\log (1+t))^{m}\right)\right) \mid x^{n-1}\right\rangle \\
& \quad+\left\langle\left(\partial_{t} \prod_{j=1}^{r}\left(\frac{(1+t)^{a_{j}}-1}{\log (1+t)}\right)\right)(\log (1+t))^{m} \mid x^{n-1}\right\rangle .
\end{aligned}
$$

Note that

$$
\begin{aligned}
& \left\langle\prod_{j=1}^{r}\left(\frac{(1+t)^{a_{j}}-1}{\log (1+t)}\right)\left(\partial_{t}\left((\log (1+t))^{m}\right)\right) \mid x^{n-1}\right\rangle \\
& \quad=m\left\langle\prod_{j=1}^{r}\left(\frac{(1+t)^{a_{j}}-1}{\log (1+t)}\right) \frac{1}{1+t} \mid \log (1+t)^{m-1} x^{n-1}\right\rangle \\
& \quad=m\left\langle\prod_{j=1}^{r}\left(\frac{(1+t)^{a_{j}}-1}{\log (1+t)}\right)(1+t)^{-1} \mid(m-1) ! \sum_{l=m-1}^{\infty} S_{1}(l, m-1) \frac{t^{l}}{l !} x^{n-1}\right\rangle \\
& \quad=m ! \sum_{l=m-1}^{n-1}\left(\begin{array}{c}
n-1 \\
l
\end{array}\right) S_{1}(l, m-1)\left\langle\prod_{j=1}^{r}\left(\frac{(1+t)^{a_{j}}-1}{\log (1+t)}\right)(1+t)^{-1} \mid x^{n-1-l}\right\rangle \\
& \quad=m ! \sum_{l=m-1}^{n-1}\left(\begin{array}{c}
n-1 \\
l
\end{array}\right) S_{1}(l, m-1) N_{n-1-l}\left(-1 \mid a_{1}, \ldots, a_{r}\right) \\
& \quad=m ! \sum_{l=0}^{n-m}\left(\begin{array}{c}
n-1 \\
l
\end{array}\right) S_{1}(n-1-l, m-1) N_{l}\left(-1 \mid a_{1}, \ldots, a_{r}\right)
\end{aligned}
$$

and

$$
\begin{aligned}
& \left\langle\left(\partial_{t} \prod_{j=1}^{r}\left(\frac{(1+t)^{a_{j}}-1}{\log (1+t)}\right)\right)(\log (1+t))^{m} \mid x^{n-1}\right\rangle \\
& =\left\langle\left(\partial_{t} \prod_{j=1}^{r}\left(\frac{(1+t)^{a_{j}}-1}{\log (1+t)}\right)\right) \mid m ! \sum_{l=m}^{\infty} S_{1}(l, m) \frac{t^{l}}{l !} x^{n-1}\right\rangle \\
& =m ! \sum_{l=m}^{n-1}\left(\begin{array}{c}
n-1 \\
l
\end{array}\right) S_{1}(l, m) \\
& \quad \times\left\langle\prod_{i=1}^{r}\left(\frac{(1+t)^{a_{i}}-1}{\log (1+t)}\right)(1+t)^{-1} \mid \frac{\sum_{j=1}^{r}\left\{\frac{a_{j} t(1+t)^{a_{j}}}{(1+t)^{a_{j}}-1}-\frac{t}{\log (1+t)}\right\}}{t} x^{n-1-l}\right\rangle \\
& =m ! \sum_{l=m}^{n-1}\left(\begin{array}{c}
n-1 \\
l
\end{array}\right) \frac{S_{1}(l, m)}{n-l}
\end{aligned}
$$




$$
\begin{aligned}
& \times\left\langle\prod_{i=1}^{r}\left(\frac{(1+t)^{a_{i}}-1}{\log (1+t)}\right)(1+t)^{-1} \mid \sum_{j=1}^{r}\left\{\frac{a_{j} t(1+t)^{a_{j}}}{(1+t)^{a_{j}}-1}-\frac{t}{\log (1+t)}\right\} x^{n-l}\right\rangle \\
= & \frac{m !}{n} \sum_{l=m}^{n-1}\left(\begin{array}{l}
n \\
l
\end{array}\right) S_{1}(l, m) \\
& \times\left\{\sum_{j=1}^{r} a_{j}\left\langle\frac{\log (1+t)}{(1+t)^{a_{j}}-1} \prod_{i=1}^{r}\left(\frac{(1+t)^{a_{i}}-1}{\log (1+t)}\right)(1+t)^{a_{j}-1} \mid \frac{t}{\log (1+t)} x^{n-l}\right\rangle\right. \\
& \left.-r\left\langle\prod_{i=1}^{r}\left(\frac{(1+t)^{a_{i}}-1}{\log (1+t)}\right)(1+t)^{-1} \mid \frac{t}{\log (1+t)} x^{n-l}\right\rangle\right\} \\
= & \frac{m !}{n} \sum_{l=m}^{n-1}\left(\begin{array}{c}
n \\
l
\end{array}\right) S_{1}(l, m)\left\{\sum_{j=1}^{r} a_{j} \sum_{k=0}^{n-l} C_{k}\left(\begin{array}{c}
n-l \\
k
\end{array}\right) N_{n-l-k}\left(a_{j}-1 \mid a_{1}, \ldots, \hat{a}_{j}, \ldots, a_{r}\right)\right. \\
& \left.-r \sum_{k=0}^{n-l} C_{k}\left(\begin{array}{c}
n-l \\
k
\end{array}\right) N_{n-l-k}\left(-1 \mid a_{1}, \ldots, a_{r}\right)\right\} .
\end{aligned}
$$

Therefore, by (55), (56), (57) and (58), we obtain the following theorem.

Theorem 6 For $n-1 \geq m \geq 1$, we have

$$
\begin{aligned}
\sum_{l=0}^{n-m}\left(\begin{array}{l}
n \\
l
\end{array}\right) S_{1}(n-l, m) N_{l}\left(a_{1}, \ldots, a_{r}\right) \\
=\sum_{l=0}^{n-m}\left(\begin{array}{c}
n-1 \\
l
\end{array}\right) S_{1}(n-l-1, m-1) N_{l}\left(-1 \mid a_{1}, \ldots, a_{r}\right) \\
\quad+\frac{1}{n} \sum_{l=m}^{n-1} \sum_{k=0}^{n-l} \sum_{j=1}^{r}\left(\begin{array}{c}
n \\
l
\end{array}\right)\left(\begin{array}{c}
n-l \\
k
\end{array}\right) a_{j} C_{n-l-k} S_{1}(l, m) N_{k}\left(a_{j}-1 \mid a_{1}, \ldots, \hat{a}_{j}, \ldots, a_{r}\right) \\
\quad-\frac{r}{n} \sum_{l=m}^{n-1} \sum_{k=0}^{n-l}\left(\begin{array}{c}
n \\
l
\end{array}\right)\left(\begin{array}{c}
n-l \\
k
\end{array}\right) C_{n-l-k} S_{1}(l, m) N_{k}\left(-1 \mid a_{1}, \ldots, a_{r}\right) .
\end{aligned}
$$

By the same method as the proof of Theorem 6, we get

$$
\begin{aligned}
\sum_{l=0}^{n-m}\left(\begin{array}{c}
n \\
l
\end{array}\right) S_{1}(n-l, m) \hat{N}_{l}\left(a_{1}, \ldots, a_{r}\right) \\
=\sum_{l=0}^{n-m}\left(\begin{array}{c}
n-1 \\
l
\end{array}\right) S_{1}(n-l-1, m-1) \hat{N}_{l}\left(-1 \mid a_{1}, \ldots, a_{r}\right) \\
\quad+\frac{1}{n} \sum_{j=1}^{r} \sum_{l=m}^{n-1} \sum_{k=0}^{n-l}\left(\begin{array}{c}
n \\
l
\end{array}\right)\left(\begin{array}{c}
n-l \\
k
\end{array}\right) a_{j} C_{n-l-k} S_{1}(l, m) \hat{N}_{k}\left(-1 \mid a_{1}, \ldots, \hat{a}_{j}, \ldots, a_{r}\right) \\
\quad-\frac{r}{n} \sum_{l=m}^{n-1} \sum_{k=0}^{n-l}\left(\begin{array}{c}
n \\
l
\end{array}\right)\left(\begin{array}{c}
n-l \\
k
\end{array}\right) C_{n-l-k} S_{1}(l, m) \hat{N}_{k}\left(-1 \mid a_{1}, \ldots, a_{r}\right) \\
\quad-\sum_{j=1}^{r} a_{j} \sum_{l=0}^{n-m-1}\left(\begin{array}{c}
n-1 \\
l
\end{array}\right) S_{1}(n-l-1, m) \hat{N}_{k}\left(-1 \mid a_{1}, \ldots, a_{r}\right),
\end{aligned}
$$

where $n-1 \geq m \geq 1$. 
Let us consider the following two Sheffer sequences:

$$
N_{n}\left(x \mid a_{1}, \ldots, a_{r}\right) \sim\left(\prod_{j=1}^{r}\left(\frac{t}{e^{a_{j} t}-1}\right), e^{t}-1\right)
$$

and (23).

We let

$$
N_{n}\left(x \mid a_{1}, \ldots, a_{r}\right)=\sum_{m=0}^{n} C_{n, m}(x)_{m}
$$

From (18) and (19), we note that

$$
\begin{aligned}
C_{n, m} & =\frac{1}{m !}\left\langle\prod_{j=1}^{r}\left(\frac{(1+t)^{a_{j}}-1}{\log (1+t)}\right) t^{m} \mid x^{n}\right\rangle \\
& =\left(\begin{array}{c}
n \\
m
\end{array}\right)\left\langle\prod_{j=1}^{r}\left(\frac{(1+t)^{a_{j}}-1}{\log (1+t)}\right) \mid x^{n-m}\right\rangle \\
& =\left(\begin{array}{c}
n \\
m
\end{array}\right) N_{n-m}\left(a_{1}, \ldots, a_{r}\right) .
\end{aligned}
$$

Therefore, by (61) and (62), we obtain the following theorem.

Theorem 7 For $n \geq 0$, we have

$$
N_{n}\left(x \mid a_{1}, \ldots, a_{r}\right)=\sum_{m=0}^{n}\left(\begin{array}{l}
n \\
m
\end{array}\right) N_{n-m}\left(a_{1}, \ldots, a_{r}\right)(x)_{m} .
$$

By the same method as the proof of Theorem 7, we get

$$
\hat{N}_{n}\left(x \mid a_{1}, \ldots, a_{r}\right)=\sum_{m=0}^{n}\left(\begin{array}{l}
n \\
m
\end{array}\right) \hat{N}_{n-m}\left(a_{1}, \ldots, a_{r}\right)(x)_{m} .
$$

For

$$
N_{n}\left(x \mid a_{1}, \ldots, a_{r}\right) \sim\left(\prod_{j=1}^{r}\left(\frac{t}{e^{a_{j} t}-1}\right), e^{t}-1\right)
$$

and

$$
H_{n}^{(s)}(x \mid \lambda) \sim\left(\left(\frac{e^{t}-\lambda}{1-\lambda}\right)^{s}, t\right), \quad \lambda \in \mathbb{C} \text { with } \lambda \neq 1
$$

let us assume that

$$
N_{n}\left(x \mid a_{1}, \ldots, a_{r}\right)=\sum_{m=0}^{n} C_{n, m} H_{m}^{(s)}(x \mid \lambda)
$$


where $H_{m}^{(s)}(x \mid \lambda)$ are the Frobenius-Euler polynomials of order $s$ defined by the generating function as

$$
\left(\frac{1-\lambda}{e^{t}-\lambda}\right)^{s} e^{x t}=\sum_{n=0}^{\infty} H_{n}^{(s)}(x \mid \lambda) \frac{t^{n}}{n !}
$$

From (18) and (19), we note that

$$
\begin{aligned}
C_{n, m}= & \frac{1}{m !(1-\lambda)^{s}}\left\langle\prod_{j=1}^{r}\left(\frac{(1+t)^{a_{j}}-1}{\log (1+t)}\right)(\log (1+t))^{m}(1-\lambda+t)^{s} \mid x^{n}\right\rangle \\
= & \frac{1}{m !(1-\lambda)^{s}}\left\langle\prod_{j=1}^{r}\left(\frac{(1+t)^{a_{j}}-1}{\log (1+t)}\right)(\log (1+t))^{m} \mid \sum_{j=0}^{\min \{s, n\}}\left(\begin{array}{l}
s \\
j
\end{array}\right)(1-\lambda)^{s-j} t^{j} x^{n}\right\rangle \\
= & \frac{1}{m !(1-\lambda)^{s}} \sum_{j=0}^{n-m}\left(\begin{array}{l}
s \\
j
\end{array}\right)(1-\lambda)^{s-j}(n)_{j} \\
& \times\left\langle\prod_{j=1}^{r}\left(\frac{(1+t)^{a_{j}}-1}{\log \left(1_{t}\right)}\right)(\log (1+t))^{m} \mid x^{n-j}\right\rangle \\
= & \frac{1}{m !(1-\lambda)^{s}} \sum_{j=0}^{n-m}\left(\begin{array}{l}
s \\
j
\end{array}\right)(1-\lambda)^{s-j}(n)_{j} m ! \\
& \times \sum_{l=0}^{n-j-m}\left(\begin{array}{c}
n-j \\
l
\end{array}\right) S_{1}(n-j-l, m) N_{l}\left(a_{1}, \ldots, a_{r}\right) \\
= & \sum_{j=0}^{n-m} \sum_{l=0}^{n-m-j}\left(\begin{array}{c}
s \\
j
\end{array}\right)\left(\begin{array}{c}
n-j \\
l
\end{array}\right) \\
& \times(n)_{j}(1-\lambda)^{-j} S_{1}(n-j-l, m) N_{l}\left(a_{1}, \ldots, a_{r}\right) .
\end{aligned}
$$

Therefore, by (64) and (65), we obtain the following theorem.

Theorem 8 For $n \geq 0$, we have

$$
\begin{aligned}
& N_{n}\left(x \mid a_{1}, \ldots, a_{r}\right) \\
& =\sum_{m=0}^{n}\left\{\sum_{j=0}^{n-m} \sum_{l=0}^{n-m-j}\left(\begin{array}{c}
s \\
j
\end{array}\right)\left(\begin{array}{c}
n-j \\
l
\end{array}\right)(n)_{j}(1-\lambda)^{-j}\right. \\
& \left.\quad \times S_{1}(n-j-l, m) N_{l}\left(a_{1}, \ldots, a_{r}\right)\right\} H_{m}^{(s)}(x \mid \lambda) .
\end{aligned}
$$

By the same method as the proof of Theorem 8 , we get

$$
\begin{aligned}
& \hat{N}_{n}\left(x \mid a_{1}, \ldots, a_{r}\right) \\
& =\sum_{m=0}^{n}\left\{\sum_{j=0}^{n-m} \sum_{l=0}^{n-m-j}\left(\begin{array}{c}
s \\
j
\end{array}\right)\left(\begin{array}{c}
n-j \\
l
\end{array}\right)(n)_{j}\right. \\
& \left.\quad \times(1-\lambda)^{-j} S_{1}(n-j-l, m) \hat{N}_{l}\left(a_{1}, \ldots, a_{r}\right)\right\} H_{m}^{(s)}(x \mid \lambda) .
\end{aligned}
$$


Now, we consider the following two Sheffer sequences:

$$
N_{n}\left(x \mid a_{1}, \ldots, a_{r}\right) \sim\left(\prod_{j=1}^{r}\left(\frac{t}{e^{a_{j} t}-1}\right), e^{t}-1\right)
$$

and

$$
B_{n}^{(s)}(x) \sim\left(\left(\frac{e^{t}-1}{t}\right)^{s}, e^{t}-1\right)
$$

where $B_{n}^{(s)}(x)$ are the Bernoulli polynomials of order $s$ given by the generating function as

$$
\left(\frac{t}{e^{t}-1}\right)^{s} e^{x t}=\sum_{n=0}^{\infty} B_{n}^{(s)}(x) \frac{t^{n}}{n !} .
$$

Let us assume that

$$
N_{n}\left(x \mid a_{1}, \ldots, a_{r}\right)=\sum_{m=0}^{n} C_{n, m} B_{m}^{(s)}(x) .
$$

By (18) and (19), we get

$$
\begin{aligned}
C_{n, m} & =\frac{1}{m !}\left\langle\prod_{j=1}^{r}\left(\frac{(1+t)^{a_{j}}-1}{\log (1+t)}\right)(\log (1+t))^{m}\left(\frac{t}{\log (1+t)}\right)^{s} \mid x^{n}\right\rangle \\
& =\frac{1}{m !}\left\langle\prod_{j=1}^{r}\left(\frac{(1+t)^{a_{j}}-1}{\log (1+t)}\right)(\log (1+t))^{m} \mid\left(\frac{t}{\log (1+t)}\right)^{s} x^{n}\right\rangle \\
& =\frac{1}{m !}\left\langle\prod_{j=1}^{r}\left(\frac{(1+t)^{a_{j}}-1}{\log (1+t)}\right)(\log (1+t))^{m} \mid \sum_{k=0}^{\infty} \mathbb{C}_{k}^{(s)} \frac{t^{k}}{k !} x^{n}\right\rangle \\
& =\frac{1}{m !} \sum_{k=0}^{n-m}\left(\begin{array}{l}
n \\
k
\end{array}\right) \mathbb{C}_{k}^{(s)}\left\langle\prod_{j=1}^{r}\left(\frac{(1+t)^{a_{j}}-1}{\log (1+t)}\right)(\log (1+t))^{m} \mid x^{n-k}\right\rangle \\
& =\frac{1}{m !} \sum_{k=0}^{n-m}\left(\begin{array}{l}
n \\
k
\end{array}\right) \mathbb{C}_{k}^{(s)} m ! \sum_{l=0}^{n-m-k}\left(\begin{array}{c}
n-k \\
l
\end{array}\right) S_{1}(n-l-k, m) N_{l}\left(a_{1}, \ldots, a_{r}\right) \\
& =\sum_{k=0}^{n-m} \sum_{l=0}^{n-m-k}\left(\begin{array}{l}
n \\
k
\end{array}\right)\left(\begin{array}{c}
n-k \\
l
\end{array}\right) \mathbb{C}_{k}^{(s)} S_{1}(n-k-l, m) N_{l}\left(a_{1}, \ldots, a_{r}\right),
\end{aligned}
$$

where $\mathbb{C}_{k}^{(s)}$ are the Cauchy numbers of the first kind of order $s$ defined by the generating function as

$$
\left(\frac{t}{\log (1+t)}\right)^{s}=\sum_{n=0}^{\infty} \mathbb{C}_{n}^{(s)} \frac{t^{n}}{n !}
$$

Therefore, by (68) and (69), we obtain the following theorem. 
Theorem 9 For $n \geq 0$, we have

$$
\begin{aligned}
N_{n}\left(x \mid a_{1}, \ldots, a_{r}\right)= & \sum_{m=0}^{n}\left\{\sum_{k=0}^{n-m} \sum_{l=0}^{n-m-k}\left(\begin{array}{l}
n \\
k
\end{array}\right)\left(\begin{array}{c}
n-k \\
l
\end{array}\right) \mathbb{C}_{k}^{(s)}\right. \\
& \left.\times S_{1}(n-k-l, m) N_{l}\left(a_{1}, \ldots, a_{r}\right)\right\} B_{m}^{(s)}(x) .
\end{aligned}
$$

By the same method as the proof of Theorem 9, we get

$$
\begin{aligned}
\hat{N}_{n}\left(x \mid a_{1}, \ldots, a_{r}\right)= & \sum_{m=0}^{n}\left\{\sum_{k=0}^{n-m} \sum_{l=0}^{n-m-k}\left(\begin{array}{l}
n \\
k
\end{array}\right)\left(\begin{array}{c}
n-k \\
l
\end{array}\right) \mathbb{C}_{k}^{(s)}\right. \\
& \left.\times S_{1}(n-k-l, m) \hat{N}_{l}\left(a_{1}, \ldots, a_{r}\right)\right\} B_{m}^{(s)}(x) .
\end{aligned}
$$

Recently, several authors have studied umbral calculus (see $[1-5,7-18,20])$.

\section{Competing interests}

The authors declare that they have no competing interests.

\section{Authors' contributions}

All authors contributed equally to the manuscript and typed, read, and approved the final manuscript.

\section{Author details}

'Department of Mathematics, Sogang University, Seoul, 121-742, Republic of Korea. ${ }^{2}$ Department of Mathematics, Kwangwoon University, Seoul, 139-701, Republic of Korea.

\section{Acknowledgements}

This work was supported by the National Research Foundation of Korea (NRF) grant funded by the Korean government (MOE) (No. 2012R1A1A2003786). The authors would like to thank the referees for their valuable comments.

Received: 30 April 2014 Accepted: 9 June 2014 Published: 22 Jul 2014

\section{References}

1. Roman, S: The Umbral Calculus. Dover, New York (2005)

2. Kim, T, Kim, DS: Some identities involving associated sequences of special polynomials. J. Comput. Anal. Appl. 16 626-642 (2014)

3. Kim, DS, Kim, T, Lee, S-H, Rim, S-H: Umbral calculus and Euler polynomials. Ars Comb. 112, $293-306$ (2013)

4. Kim, DS, Kim, T: Some identities of Bernoulli and Euler polynomials arising from umbral calculus. Adv. Stud. Contemp. Math. (Kyungshang) 23(1), 159-171 (2013)

5. Kim, DS, Kim, T, Dolgy, DV, Rim, S-H: Some new identities of Bernoulli, Euler and Hermite polynomials arising from umbral calculus. Adv. Differ. Equ. 2013, 73 (2013)

6. Kim, T, Mansour, T, Rim, S-H, Lee, S-H: Apostol-Euler polynomials arising from umbral calculus. Adv. Differ. Equ. 2013, $301(2013)$

7. Kim, DS, Kim, T: Higher-order Cauchy of the first kind and poly-Cauchy of the first kind mixed type polynomials. Adv. Stud. Contemp. Math. (Kyungshang) 23(21), 621-636 (2013)

8. Kim, T, Kim, DS, Mansour, T, Rim, S-H, Schork, M: Umbral calculus and Sheffer sequences of polynomials. J. Math. Phys. 54(8), $083504(2013)$

9. Kim, T: Identities involving Laguerre polynomials derived from umbral calculus. Russ. J. Math. Phys. 21(1), 36-45 (2014)

10. Lu, D-Q, Xiang, Q-M, Luo, C-H: Some results for Apostol-type polynomials associated with umbral algebra. Adv. Differ. Equ. 2013, 201 (2013)

11. Maldonado, M, Prada, J, Senosiain, MJ: Appell bases on sequence spaces. J. Nonlinear Math. Phys. 18, suppl. 1, 189-194 (2011)

12. Araci, S, Kong, $X$, Acikgoz, M, Şen, E: A new approach to multivariate $q$-Euler polynomials using the umbral calculus. J. Integer Seq. 17(1), Article 14.1.2 (2014)

13. Dere, R, Simsek, Y: Applications of umbral algebra to some special polynomials. Adv. Stud. Contemp. Math. (Kyungshang) 22(3), 433-438 (2012)

14. Ernst, T: Examples of a q-umbral calculus. Adv. Stud. Contemp. Math. (Kyungshang) 16(1), 1-22 (2008)

15. Fang, Q, Wang, T: Umbral calculus and invariant sequences. Ars Comb. 101, 257-264 (2011)

16. Gessel, IM: Applications of the classical umbral calculus. Dedicated to the memory of Gian-Carlo Rota. Algebra Univers. 49(4), 397-434 (2003) 
17. Kim, DS, Kim, T: Some identities of Frobenius-Euler polynomials arising from umbral calculus. Adv. Differ. Equ. 2012, $196(2012)$

18. Kim, DS, Kim, T, Ryoo, CS: Sheffer sequences for the powers of Sheffer pairs under umbral composition. Adv. Stud. Contemp. Math. (Kyungshang) 23(2), 275-285 (2013)

19. Ryoo, CS, Song, H, Agarwal, RP: On the roots of the $q$-analogue of Euler-Barnes' polynomials. Adv. Stud. Contemp. Math. 9(2), 153-163 (2004)

20. Taylor, BD: Umbral presentations for polynomial sequences. Umbral calculus and its applications (Cortona, 1998). Comput. Math. Appl. 41(9), 1085-1098 (2001)

10.1186/1687-1847-2014-182

Cite this article as: Kim and Kim: Barnes-type Narumi polynomials. Advances in Difference Equations 2014, 2014:182

Submit your manuscript to a SpringerOpen ${ }^{\odot}$ journal and benefit from:

- Convenient online submission

- Rigorous peer review

- Immediate publication on acceptance

- Open access: articles freely available online

- High visibility within the field

- Retaining the copyright to your article

Submit your next manuscript at $\boldsymbol{s p r i n g e r o p e n . c o m ~}$ 